\title{
Exigência Nutricional de Cálcio para Frangos de Corte, nas Fases de Crescimento e Terminação ${ }^{1}$
}

\section{Luciano Moraes Sá2 ${ }^{\text {, Paulo Cezar Gomes }}{ }^{3}$, Horacio Santiago Rostagno ${ }^{3}$, Luiz Fernando Teixeira Albino ${ }^{3}$, Paulo Roberto Cecon ${ }^{4}$, Priscila D'Agostini ${ }^{2}$}

\begin{abstract}
RESUMO - Foram conduzidos dois experimentos com o objetivo de determinar a exigência nutricional de cálcio para frangos de corte de 22 a 42 e 43 a 53 dias de idade. Em cada experimento foram utilizados 360 frangos de corte da linhagem Avian Farm, sendo 180 machos e 180 fêmeas. O primeiro ensaio teve duração de 21 dias e o segundo, de 10 dias, sendo que em ambos o delineamento experimental adotado foi o inteiramente casualizado, com seis tratamentos e seis repetições, sendo a unidade experimental representada pelo box, contendo cinco machos e cinco fêmeas. Foram formuladas duas dietas basais constituídas de milho e farelo de soja, suplementadas com vitaminas e minerais, atendendo as exigências nutricionais das aves, com exceção do cálcio, que permaneceu deficiente no nível de $0,16 \%$. Os tratamentos, em cada experimento, foram constituídos da suplementação das dietas basais com 0,0;0,25;0,50;0,75; 1,00; e 1,25\% de cálcio provenientes do carbonato de cálcio, resultando nos níveis de 0,$16 ; 0,41 ; 0,66 ; 0,91 ; 1,16$; e 1,41\% de cálcio. Com o término de cada experimento, foram abatidas 72 aves (duas aves por box), para extração das tíbias, visando à medição da resistência à quebra e análises de cálcio e cinzas nos ossos. Os valores da exigência de cálcio foram estimados por intermédio de ganho de peso, conversão alimentar, teores de cálcio e cinzas nos ossos (em porcentagem e em gramas) e resistência à quebra dos ossos, utilizando-se os modelos de regressão linear e/ou quadrática. De acordo com as respostas biológicas das aves frente aos níveis de cálcio estudados, sugerem-se os valores de 1,02 e 1,01\% de cálcio como a exigência para frangos de corte de 22 a 42 e 43 a 53 dias de idade, respectivamente. Contudo, levando-se em consideração a resistência à quebra óssea, a exigência de cálcio para frangos de corte de 22 a 42 e 43 a 53 dias de idade seria de 1,28 e $1,18 \%$, respectivamente.
\end{abstract}

Palavras-chave: requerimento de cálcio, desempenho, ossos, frango de corte

\section{Calcium Requirement for Broiler Chicks from 22 to 42 and 43 to 53 Days Old}

ABSTRACT - Two experiments were carried out to determine the nutritional requirement of calcium for broilers in growing (22 to 42 days), and finishing (43 to 53 days) phases. Three hundred and sixty Avian Farm broiler chicks (180 from each sex) were used in both trails. The first experiment lasted 21 days and the second one, 10 days. A completely randomized design was used in both experiments, with six treatments, six replications and 10 birds per experimental unity (five males and five females). Two basal diets were formulated to meet the birds nutritional requirements, except for the calcium, that remained deficient at the level of $0.16 \%$. The basal diets in each experiment were supplemented with $0.0,0.25,0.50,0.75,1.00$, and $1.25 \%$ calcium from calcium carbonate, resulting in the levels of $0.16,0.41,0.66,0.91,1.16$ and $1.41 \%$ of calcium, and the treatments were used to estimated the calcium requirements of broilers. At the end of each experiment, 72 birds were sacrificed by cervical dislocation for the purpose of determining the tibia breaking strength and tibia obtained calcium and ash. Weight gain, feed:gain ratio, tibia calcium contents (percentage and gram), tibia breaking strength (kgf), ashes contents in the tibia (percentage and gram) were the evaluated variables. The calcium requirements were estimated using the quadratic regression model. According to the biological values observed for the chickens were suggested the calcium requirements estimates of 1.02 and $1.01 \%$ for broilers from 22 to 42 and 43 to 53 days old, respectively. However, according to the tibia breaking strength, the calcium requirement suggested for broilers from 22 to 42 and 43 to 53 days old would be 1.28 and $1.18 \%$ respectively.

Key Words: calcium requirements, performance, bones, broiler chicks

\section{Introdução}

O cálcio e o fósforo são minerais importantes na nutrição animal, por serem necessários não apenas para a ótima taxa de crescimento, mas também para a mineralização óssea, e estarem envolvidos em grande número de processos fisiológicos, como a transmissão de impulsos nervosos, contração muscular, coagulação sangüínea e ativação de sistemas enzimáticos. Nas aves, o cálcio é o mineral encontrado

\footnotetext{
${ }_{1}^{1}$ Parte da tese de Mestrado do primeiro autor apresentada à Universidade Federal de Viçosa.

2 Estudante de Doutorado/UFV.

3 Professor do DZO/UFV.

4 professor do DPI/UFV.
} 
em maior quantidade, estando presente quase que em sua totalidade (99\%) no esqueleto ósseo, sendo requerido em quantidade maior que qualquer outro mineral. Segundo Klasing (1998), o cálcio é o mineral metabolicamente mais ativo, com um complexo sistema regulatório.

De acordo com Smith \& Kabaija (1984), o nível de cálcio contido nos ingredientes da ração deve ser considerado na estimativa do requerimento desses animais. Segundo Scheideler et al. (1995), frangos de corte conseguem ganhar peso eficientemente com relativa amplitude de variação dos níveis de cálcio e fósforo dietéticos.

Grande número de produtos é utilizado em todo mundo como fonte de cálcio e fósforo em dietas para aves. Fontes com elevada concentração de cálcio incluem rochas calcárias, conchas calcárias e algas calcárias. Existem também várias fontes naturais como fosfatos de rocha, depósitos de guano, farinha de carne e/ou ossos. Entretanto, a maioria dos fosfatos utilizados na alimentação avícola constitui-se em produtos quimicamente processados. $\mathrm{O}$ grupo dos fosfatos bicálcicos é produzido por intermédio da reação do ácido fosfórico com calcário, originando os fosfatos monocálcicos e bicálcicos nestes processos químicos.

A importância do cálcio e fósforo na nutrição das aves pode ser demonstrada pelo vasto número de pesquisas enfocando estes minerais. Os primeiros trabalhos indicam que os requerimentos de cálcio propostos para uma taxa normal de crescimento para frangos de corte são de 0,5\% (Simco \& Stephson, 1961); 0,6\% (White-Stevens et al., 1960; Formica et al., 1962); 0,6 a 0,72\% (Waldroup et al., 1962); e 0,99 e 1,07\% (Edwards et al., 1963).

Segundo Cabral (1999), a falta de concordância entre os pesquisadores sobre os níveis adequados de cálcio pode ser atribuída a vários fatores ambientais, nutricionais, genéticos e de manejo, que influem na exigência desse mineral pelas aves, devendo-se ressaltar que diferentes metodologias são utilizadas na determinação das exigências nutricionais de cálcio. De acordo com Hurwitz et al. (1995), as respostas dos frangos de corte ao nível de cálcio dietético são marcantemente modificadas pela taxa de crescimento das aves, determinada por seu potencial genético.

Em razão do crescente avanço no melhoramento genético dos frangos de corte, ocorre constante aumento na taxa de crescimento dessas aves, tornando-se necessária a realização de pesquisas para reavaliar suas necessidades nutricionais. Devido ao pequeno número de trabalhos científicos sobre a exigência de cálcio, para frangos de corte nas fases de crescimento (3 a 6 semanas) e terminação (6 a 8 semanas), principalmente para as modernas linhagens, é interessante reavaliar o requerimento desse mineral.

O objetivo deste experimento foi estabelecer a exigência nutricional de cálcio para frangos de corte nas fases de 22 a 42 dias de idade (crescimento) e de 43 a 53 dias de idade (terminação).

\section{Material e Métodos}

Dois experimentos, sendo experimento 1 de 22 a 42 dias e experimento 2 de 43 a 53 dias de idade dos frangos, foram conduzidos no Setor de Avicultura do Departamento de Zootecnia da Universidade Federal de Viçosa - UFV, nos períodos de 21 de setembro a 11 de outubro (experimento 1 ) e de 19 a 30 de outubro de 2000 (experimento 2).

Em cada experimento foram utilizados 360 pintos de corte Avian Farm, metade machos e metade fêmeas, com 22 dias de idade no experimento 1 e com 42 dias de idade no experimento 2, todos vacinados contra Bouba Aviária e doença de Marek no incubatório. No período pré-experimental, as aves receberam ração à base de milho e farelo de soja, atendendo às recomendações nutricionais de Rostagno et al. (2000), até 21 dias de idade (experimento 1) e até 42 dias de idade (experimento 2), quando foram transferidas para os boxes. $O$ primeiro experimento teve duração de 21 dias e o segundo, de 10 dias; sendo que o delineamento experimental adotado em ambos experimentos foi o inteiramente casualizado, com seis tratamentos, seis repetições, sendo a unidade experimental representada pelo box, contendo cinco machos e cinco fêmeas.

As aves foram alojadas em boxes de 2,10 x 1,00 m, com piso de cimento, cobertos com cerca de $5 \mathrm{~cm}$ de cepilho de madeira, em um galpão de alvenaria, com dimensões de 29,20 x 12,60 m, 2,80 m de pé-direito, coberto com telha de cimento amianto, com lanternim, laterais possuindo muretas de $0,65 \mathrm{~m}$ de altura e o restante fechado com tela de meia polegada e cortinas plásticas com abertura de baixo para cima.

O programa de luz adotado em ambos os experimentos foi o contínuo, com 24 horas de luz (natural + artificial), durante todo o período experimental, utilizando-se lâmpadas de $60 \mathrm{~W}$. A temperatura no interior do galpão foi medida diariamente por intermédio 
Tabela 1 - Composição percentual da dieta basal, para as fases de crescimento (experimento 1) e terminação (experimento 2)

Table 1 - Composition of the basal diet (\%) for the growing (experiment 1) and finishing (experiment 2) phases

\begin{tabular}{|c|c|c|}
\hline $\begin{array}{l}\text { Ingredientes } \\
\text { Ingredients }\end{array}$ & $\begin{array}{c}\text { Crescimento \% } \\
\text { Growing }\end{array}$ & $\begin{array}{c}\text { Terminação \% } \\
\text { Finishing }\end{array}$ \\
\hline Milho (Corn) & 58,00 & 60,73 \\
\hline Farelo de soja (Soybean meal) & 33,59 & 29,85 \\
\hline Óleo vegetal (Vegetal oil) & 2,80 & 4,00 \\
\hline Fosfato monoamônio (Monoammonium phosphate) & 1,20 & 1,05 \\
\hline Calcário(Limestone) & 0,13 & 0,16 \\
\hline $\operatorname{Sal}($ Salt $)$ & 0,40 & 0,39 \\
\hline Bacitracina de zinco (Zinc bacitracin) & 0,02 & 0,02 \\
\hline Cloreto de colina (60\%) (Choline chloride) & 0,10 & 0,10 \\
\hline Anticoccidiano (Anticoccidial agent $)^{1}$ & 0,10 & 0,10 \\
\hline DL-metionina (99\%) (DL-methionine) & 0,20 & 0,14 \\
\hline L- lisina $(78 \%)$ & 0,10 & 0,10 \\
\hline BHT & 0,01 & 0,01 \\
\hline Mistura mineral (Mineral mix $)^{2}$ & 0,05 & 0,05 \\
\hline Mistura vitamínica (Vitamin mix) ${ }^{3}$ & 0,10 & 0,10 \\
\hline Caulin (Inert) & 3,20 & 3,20 \\
\hline Total & 100 & 100 \\
\hline \multicolumn{3}{|l|}{$\begin{array}{l}\text { Composição calculada } \\
\text { Calculated composition }\end{array}$} \\
\hline Proteína bruta (\%) (Crude protein) & 19,52 & 18,50 \\
\hline Energia metabolizável (Mcal/kg) & 3,100 & 3,202 \\
\hline \multicolumn{3}{|l|}{ Metabolizable energy } \\
\hline Cálcio $(\%)($ Calcium $)$ & 0,161 & 0,161 \\
\hline Fósforo disponível (\%) (Available phosphorus) & 0,410 & 0,368 \\
\hline Metionina (\%) (Methionine) & 0,515 & 0,436 \\
\hline $\operatorname{Lisina}(\%)($ Lysine $)$ & 1,156 & 1,060 \\
\hline Metionina + Cistina $(\%)($ Methionine + Cystine $)$ & 0,839 & 0,742 \\
\hline Treonina $(\%)$ (Threonine) & 0,865 & 0,811 \\
\hline Triptofano (\%) (Thryptophan) & 0,300 & 0,279 \\
\hline Sódio $(\%)$ (Sodium) & 0,199 & 0,193 \\
\hline
\end{tabular}

${ }^{1}$ Anticoccidiano - (20\% de monensina sódica).

${ }^{2}$ Níveis de garantia/kg do produto (Guarantee levels/kg of product): Fe, 100,0 g; Co 2,0 g; Cu 20,0 g; Mn 106,0 g; I 2,0 g; e veículo gsp $1000 \mathrm{~g}$.

${ }^{3}$ Níveis de garantia/kg do produto (Guarantee levels/kg of product): vit. A, 10.000.000 UI; vit. D3, 2.000.000 UI; vit. E, 30.000 UI; vit. B, 2,0 g; vit. B6, 3,0 g; ác. pantotênico (panthotenic acid), 12,0 g; biotina (biotin), 0,1 g; vit. K3, 3,0 g; ác. fólico, 1,0 g; ác. nicotínico (nicotinic acid), 50,0 g; bacitracina de Zn (zinc bacitracin), 10,0g; BHT, 5,0 g; vit. B12, 15000 mcg; Se, 0,25 g e veículo gsp $1000 \mathrm{~g}$.

de termômetros de máxima e mínima espalhados pelo interior do galpão, colocados ao nível do dorso dos animais, durante ambos os ensaios.

As composições químicas dos ingredientes utilizados na formulação das dietas foram obtidas através de análises realizadas no Laboratório de Nutrição Animal do Departamento de Zootecnia da UFV. As concentrações de cálcio determinadas foram de 0,$03 ; 0,267$; 0,46 e $39,65 \%$ para o milho, farelo de soja, fosfato monoamônio e carbonato de cálcio, respectivamente. Os teores de matéria seca e proteína bruta foram de 87,11 e 7,80\%, respectivamente, para o milho, e de
87,74 e 44,0\%, respectivamente, para o farelo de soja.

A dieta basal foi formulada utilizando-se milho e farelo de soja suplementada com vitaminas e minerais (Tabela 1), atendendo às exigências nutricionais das aves, como preconizado por Rostagno et al. (2000), com exceção do cálcio, que permaneceu deficiente no nível de $0,16 \%$.

Os tratamentos em ambos experimentos foram constituídos da suplementação da dieta basal com 0,$0 ; 0,25 ; 0,50 ; 0,75 ; 1,00 ;$ e $1,25 \%$ de cálcio proveniente do carbonato de cálcio, totalizando os níveis de 0,16 ; 0,$41 ; 0,66 ; 0,91 ; 1,16 ;$ e $1,41 \%$ de cálcio. 
As aves receberam ração e água à vontade; as pesagens foram realizadas no início e final de cada experimento para averiguação do ganho de peso, consumo de ração e conversão alimentar. Com o término de cada experimento, foram abatidas 72 aves com o peso médio do box (duas aves por box), para extração das tíbias, visando à medição da resistência à quebra e análises de cinzas e cálcio.

As tíbias direitas foram destinadas para o teste de resistência à quebra, e as esquerdas, após a retirada de gordura no extrator "Soxhlet", colocadas na mufla a $600^{\circ} \mathrm{C}$ durante quatro horas para determinação das cinzas. O teor de cálcio nos ossos foi obtido de acordo com a metodologia proposta por Silva (1998). As análises de resistência à quebra óssea foram realizadas utilizando-se os ossos in natura, em uma prensa computadorizada, que registra a resistência de materiais (Instron Corporation Series IX Automated Materials Testing System $1,09)$. Os ossos foram colocados na posição horizontal sobre dois suportes, sendo a pressão aplicada no centro dos mesmos. A quantidade máxima de força aplicada ao osso antes de sua ruptura foi considerada como resistência à quebra.

Os valores da exigência de cálcio foram estimados por intermédio de ganho de peso, conversão alimentar, teores de cálcio e cinzas nos ossos (em porcentagem e em gramas) e resistência à quebra dos ossos, utilizando-se os modelos de regressão linear e/ou quadrática, conforme ajuste dos dados obtidos para cada variável, interpretando-se as respostas biológicas das aves. A escolha entre os modelos foi feita pela observação dos coeficientes de regressão e pela soma de quadrados dos desvios, nos quais foram privilegiados os maiores coeficientes e as menores somas de quadrado. De acordo com Euclydes \& Rostagno (2001), as exigências determinadas pelo modelo quadrático geralmente são superestimadas; assim, optou-se pela utilização de $95 \%$ do máximo valor encontrado para cada exigência, de acordo com Kidd et al. (1999). As variáveis acima citadas foram consideradas dependentes e os níveis de cálcio em estudo, variáveis independentes, na análise de regressão.

As análises estatísticas dos parâmetros avaliados neste experimento foram realizadas de acordo com o programa SAEG (Sistema para Análises Estatísticas e Genéticas), (versão 7.0) desenvolvido pela Universidade Federal de Viçosa, 1997.

\section{Resultados e Discussão}

\section{Experimento 1 - Exigência nutricional de cálcio para frangos de corte no perío- do de crescimento $(22$ a 42 dias de idade)}

A temperatura máxima média e mínima média observada foi de 28,38 e $19,23^{\circ} \mathrm{C}$, respectivamente. A variação da temperatura, por estar dentro da zona de conforto dos frangos, provavelmente não interferiu nos resultados deste experimento.

Os resultados de desempenho e dos parâmetros ósseos observados com frangos de corte de 22 a 42 dias de idade estão apresentados na Tabela 2.

O ganho de peso (GP) foi influenciado pelos níveis de cálcio estudados, sendo o menor ganho obtido com o nível de $0,16 \%$ de cálcio e o maior, com $0,91 \%$ de cálcio na dieta. Pode-se observar que houve pequena variação na taxa de ganho de peso, à medida que se elevou o nível de cálcio de 0,41 a $1,41 \%$.

A conversão alimentar (CA) e o consumo de ração $(\mathrm{CR})$ não foram influenciados pelos níveis de cálcio estudados neste experimento.

De acordo com o modelo de regressão quadrática, a exigência de cálcio estimada pelo GP foi de $0,93 \%$ (Tabela 3). Esse valor foi superior àquele recomendado por Rostagno et al. (2000), que sugerem o nível de $0,874 \%$ de cálcio, e pelo NRC (1994), que sugere o nível de $0,9 \%$ de cálcio na dieta para frangos de corte em crescimento. Entretanto, Hulan et al. (1985) recomendam $1,3 \%$ de cálcio e $0,68 \%$ de fósforo para frangos de corte em fase de crescimento. Anderson et al. (1984) verificaram que o desempenho e o teor de cinzas ósseas de frangos de corte reduziram significativamente, devido ao aumento do cálcio dietético de 0,9 para $1,5 \%$.

Com exceção do teor de cálcio no osso em gramas (CAOG), as variáveis ósseas foram influenciadas pelos níveis de cálcio estudados. Houve pouca variação na porcentagem de cálcio nos ossos (CAOP) e no teor de cinza nos ossos, em porcentagem (COP) e gramas (COG), nos tratamentos em que as aves receberam os níveis de 0,66 a $1,41 \%$ de cálcio na dieta. Os menores valores para essas variáveis foram obtidos nos níveis de 0,16 e $0,41 \%$ de cálcio na dieta, em relação aos outros tratamentos.

Observaram-se maiores valores e pequena variação 


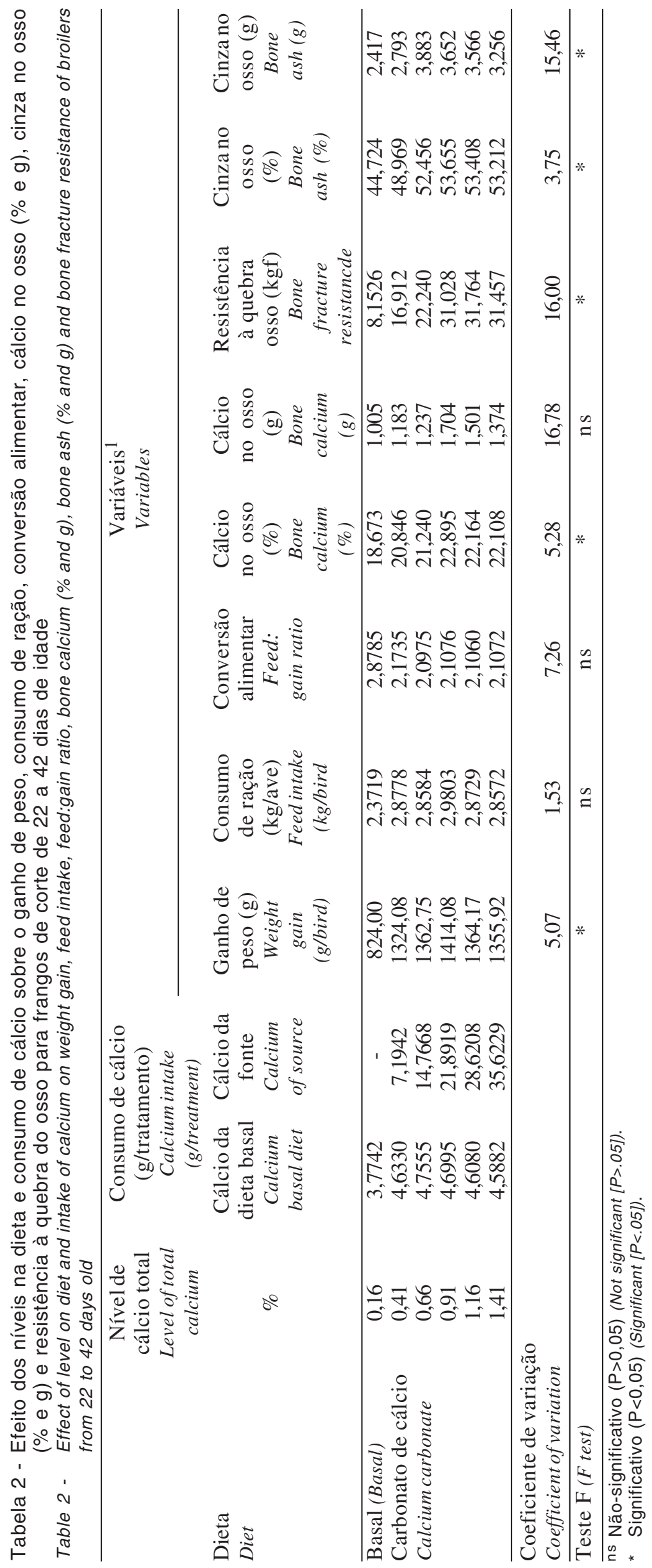


entre os resultados de resistência à quebra óssea (RQO) nos tratamentos com 0,$91 ; 1,16$; e $1,41 \%$ de cálcio, sendo superiores aos valores numéricos nos demais tratamentos. O maior valor de resistência à quebra óssea foi observado no tratamento com $1,16 \%$ de cálcio.

Estimando os valores das exigências de cálcio pelo modelo de regressão quadrática, para as variáveis CAOP, RQO, COP e COG, encontraram-se os valores de 1,$01 ; 1,28 ; 1,01 ;$ e $0,95 \%$, respectivamente (Tabela 3). Cabral (1999) verificou que a deposição de cálcio no fêmur de frangos de corte na fase de 22 a 42 dias de idade foi maior, quando os machos receberam $0,93 \%$ de cálcio na dieta e as fêmeas $0,82 \%$. Com relação à deposição de cálcio em porcentagem na tíbia, esse autor não verificou influência dos níveis de cálcio na dieta de machos e fêmeas.

Guinotte et al. (1991) observaram que frangos de corte na fase de crescimento apresentaram maior resistência à quebra do osso, quando alimentados com $0,9 \%$ de cálcio na dieta, sendo este valor menor do que o obtido neste experimento $(1,28 \%)$. Já Skinner et al. (1992) constataram que houve máxima resistência da tíbia em frangos que consumiram $0,6 \%$ de cálcio na dieta.

Neste experimento, o nível de 1,01\% de cálcio proporcionou maior deposição de cinzas nos ossos, sendo este valor superior ao verificado por Cabral (1999), o qual observou máxima deposição de cinzas em fêmures de frangos de corte machos alimentados com $0,7 \%$ de cálcio. Por outro lado, McNaughton et al. (1974) sugerem o nível de 0,8\% de cálcio para maximizar a deposição das cinzas ósseas.

As estimativas de exigência de cálcio para frangos de corte de 22 a 42 dias de idade variaram de 0,$93 ; 1,01$; 1,$28 ; 1,01$; e 0,95 para as variáveis GP, CAOP, RQO, COP e COG, respectivamente, pelo modelo quadrático. Pode-se observar que o requerimento para suprir a exigência de desempenho foi inferior aos requerimentos para as variáveis ósseas. Fato semelhante foi observado por Runho et al. (2001) e Brugalli (1996), trabalhando com exigência de fósforo disponível para frangos de corte, e por Cabral (1999), trabalhando com exigência de cálcio.

\section{Experimento 2 - Exigência nutricional de cálcio para frangos de corte no perío- do de terminação (43 a 53 dias de idade)}

Os resultados de desempenho e parâmetros ósseos observados com frangos de corte de 43 a 53 dias de idade são apresentados na Tabela 4.

Tabela 3 - Estimativas da exigência de cálcio para frangos de corte na fase de crescimento (22 a 42 dias de idade), considerando a taxa de ganho de peso, o cálcio no osso (\%), a resistência à quebra dos ossos e a cinza no osso (\% e g), ajustada por meio do modelo de regressão

Table 3 - Estimates of calcium requirement for broiler in the growing phase (from 22 to 42 days old), considering weight gain, bone calcium (\%), bone fracture resistance, and bone ash (\% and $\mathrm{g})$, adjusted by the regression model

\begin{tabular}{llcc}
\hline $\begin{array}{l}\text { Modelo } \\
\text { Model }\end{array}$ & $\begin{array}{l}\text { Equação de regressão } \\
\text { Regression equation }\end{array}$ & $\begin{array}{c}\text { Pmáx/ } \\
\text { PMín }\end{array}$ & $\begin{array}{c}\text { Exigência } \\
\text { cálcio }^{1}(\%) \\
\text { Calcium }^{2} \\
\text { requirement }{ }^{1}\end{array}$ \\
\hline
\end{tabular}

Quadrático

Quadratic

\begin{tabular}{|c|c|c|c|c|c|}
\hline $\begin{array}{l}\text { Ganho de peso } \\
\text { Weight gain }\end{array}$ & $\hat{Y}=659,5+1624,27 x-827,421 x 2$ & 1454,43 & 0,93 & $0,87 *$ & 32998,35 \\
\hline Cálcio no osso (\%) & $\hat{\mathrm{Y}}=17,3278+9,63131 \mathrm{x}-4,47077 \mathrm{x} 2$ & 22,495 & 1,01 & $0,93 *$ & 0,72250 \\
\hline $\begin{array}{l}\text { Bone calcium (\%) } \\
\text { Resistência à quebra (kgf) }\end{array}$ & $\hat{Y}=1,57367+45,2892 x-16,7615 x 2$ & 32,082 & 1,28 & $0,98 * *$ & 9,18700 \\
\hline $\begin{array}{l}\text { Bone fracture resistance }(\mathrm{kgf}) \\
\text { Cinza no osso }(\%) \\
\text { Bone ash }(\%)\end{array}$ & $\hat{Y}=41,3329+23,1909 x-10,6119 x 2$ & 53,931 & 1,01 & $0,99 * *$ & 0,57868 \\
\hline $\begin{array}{l}\text { Cinza no osso }(\mathrm{g}) \\
\text { Bone ash }(\mathrm{g})\end{array}$ & $\hat{Y}=1,80434+3,53727 x-1,75726 x 2$ & 3,5788 & 0,95 & $0,96 * *$ & 0,07569 \\
\hline
\end{tabular}

${ }_{1}^{1}$ Representa 95\% do valor máximo encontrado pela equação de regressão, indicando o nível adequado de cálcio total para cada variável. ${ }^{1}$ Represent $95 \%$ of the minimum value obtained by the regression equation, indicating the ideal total calcium levels for each levels.

** $(\mathrm{P}<.01),{ }^{*}(\mathrm{P}<.05)$ pelo teste $\mathrm{F}$.

Pmáx (ponto de máxima) e Pmín (ponto de mínima).

SQD - Soma de quadrados dos desvios (Sum of deviation squares).

\section{R. Bras. Zootec., v.33, n.2, p.397-406, 2004}


Os valores de ganho de peso (GP) e conversão alimentar (CA) e consumo de ração (CR) não foram influenciados pelos níveis de cálcio em estudo (Tabela 5). Skinner et al. (1992) e Cabral (1999), trabalhando com níveis de 0,6 a $1,2 \%$ e 0,9 a $1,3 \%$ de cálcio, respectivamente, também observaram pouca influência desses níveis de cálcio no desempenho de frangos de corte de 43 a 53 dias de idade.

Resultados semelhantes foram observados por Skinner \& Waldroup (1992), que, ao trabalharem com frangos de corte na fase de terminação não encontraram efeito da retirada da fonte de cálcio no desempenho das aves. Segundo Pinheiro et al. (1995), a retirada do calcário e/ou fosfato bicálcico da ração não prejudicou o desempenho das aves no período de 38 a 45 dias. O fato de não terem sido observadas diferenças significativas no desempenho das aves durante esta fase pode estar relacionado à possível mobilização do cálcio do tecido ósseo, que poderia estar sendo utilizado para suprir os requerimentos dos frangos em relação a este mineral.

Os valores obtidos para as variáveis cálcio no osso, em porcentagem (CAOP) e em gramas (CAOG), e para cinza no osso, em percentagem (COP) e em gramas (COG), foram semelhantes quando as aves receberam de 0,66 a 1,41\% de cálcio na dieta, sendo verificados valores inferiores quando os níveis de cálcio foram de 0,16 a $0,41 \%$. Para a resistência à quebra do osso (RQO), os valores elevaram-se com o aumento do cálcio na dieta até o nível de $1,16 \%$.

De acordo com o modelo de regressão quadrática, as exigências de cálcio estimadas variaram de $0,91 \%$ para CAOP e 1,18\% para RQO (Tabela 5). Com relação ao teor de cálcio no osso, Cabral (1999) reporta que houve máxima deposição deste mineral com o nível de $0,92 \%$ de cálcio dietético para machos e $0,93 \%$ para fêmeas. Porém, Hulan et al. (1985) recomendam $1,29 \%$ de cálcio e $0,51 \%$ de fósforo para maior deposição de minerais no osso de frangos de corte na fase de terminação. Entretanto, Edwards \& Veltmann (1983) reportam que a retenção de cálcio foi maior nos tratamentos que continham menor teor de cálcio dietético.

Excluindo-se a exigência necessária para maximizar a resistência à quebra óssea, que foi de $1,18 \%$, os valores de requerimento de cálcio encontrados neste trabalho foram superiores aos recomendados por Rostagno et al. (1996), que sugerem 0,871\% de cálcio para frangos de corte na fase de terminação, e Rostagno et al. (2000) e NRC (1994), que recomendam o valor de $0,80 \%$ para aves nesta mesma fase.

Skinner et al. (1992) também observaram a influência dos níveis de cálcio na dieta sobre a resistência da tíbia à quebra, obtendo resistência máxima com o nível de $0,6 \%$ de cálcio. Skinner \& Waldroup (1992) observaram que houve menor resistência à quebra óssea, quando as aves, no período de 42 a 49 dias de idade, receberam níveis baixos de cálcio. Entretanto, Cabral (1999) relatou que a resistência da tíbia à quebra não foi influenciada pelo nível dietético de cálcio ( 0,9 a 1,3\%) em frangos de corte de 43 a 53 dias de idade, fato não observado neste experimento, em que o nível de $1,18 \%$ de cálcio proporcionou maior valor de resistência à quebra óssea.

Neste experimento, a COP elevou-se com o aumento de cálcio na dieta, concordando com os resultados encontrados por Hulan et al. (1985), Twning et al. (1964), Waldroup et al. (1974) e Huyghebaert et al. (1981). Yoshida \& Hoshii (1982) reportaram que $1,1 \%$ de cálcio e $0,35 \%$ de fósforo são necessários para otimizar a deposição de cinzas nos ossos, em frangos de corte de 4 a 8 semanas de idade. Contudo, Cabral (1999) reportou que o teor de cinzas no fêmur foi afetado pelo nível de cálcio dietético apenas para frangos de corte machos, no período de 43 a 53 dias de idade. Segundo esse autor, houve decréscimo no teor de cinza, à medida que se elevou o nível de cálcio na ração, atingindo o teor máximo de cinza com o nível de $0,7 \%$ de cálcio, enquanto, neste experimento, o nível de $0,97 \%$ de cálcio promoveu maior deposição óssea.

As estimativas de exigência de cálcio para frangos de corte de 43 a 53 dias de idade foram de 0,$91 ; 0,94 ; 1,18$; e $0,97 \%$, para as variáveis CAOP, CAOG, RQO e COP, respectivamente, pelo modelo quadrático.

\section{Conclusões}

De acordo com as respostas biológicas das aves frente aos níveis de cálcio estudados, sugere-se os valores de 1,01 e $0,97 \%$ de cálcio como sendo a exigência para frangos de corte nos períodos de 22 a 42 e 43 a 53 dias de idade, respectivamente. Os valores sugeridos são em função do atendimento da exigência dos parâmetros cálcio no osso (\%) e cinza no osso (g) para a fase de crescimento e cinza no osso (\%) para a fase de terminação. Contudo, levando-se

R. Bras. Zootec., v.33, n.2, p.397-406, 2004 


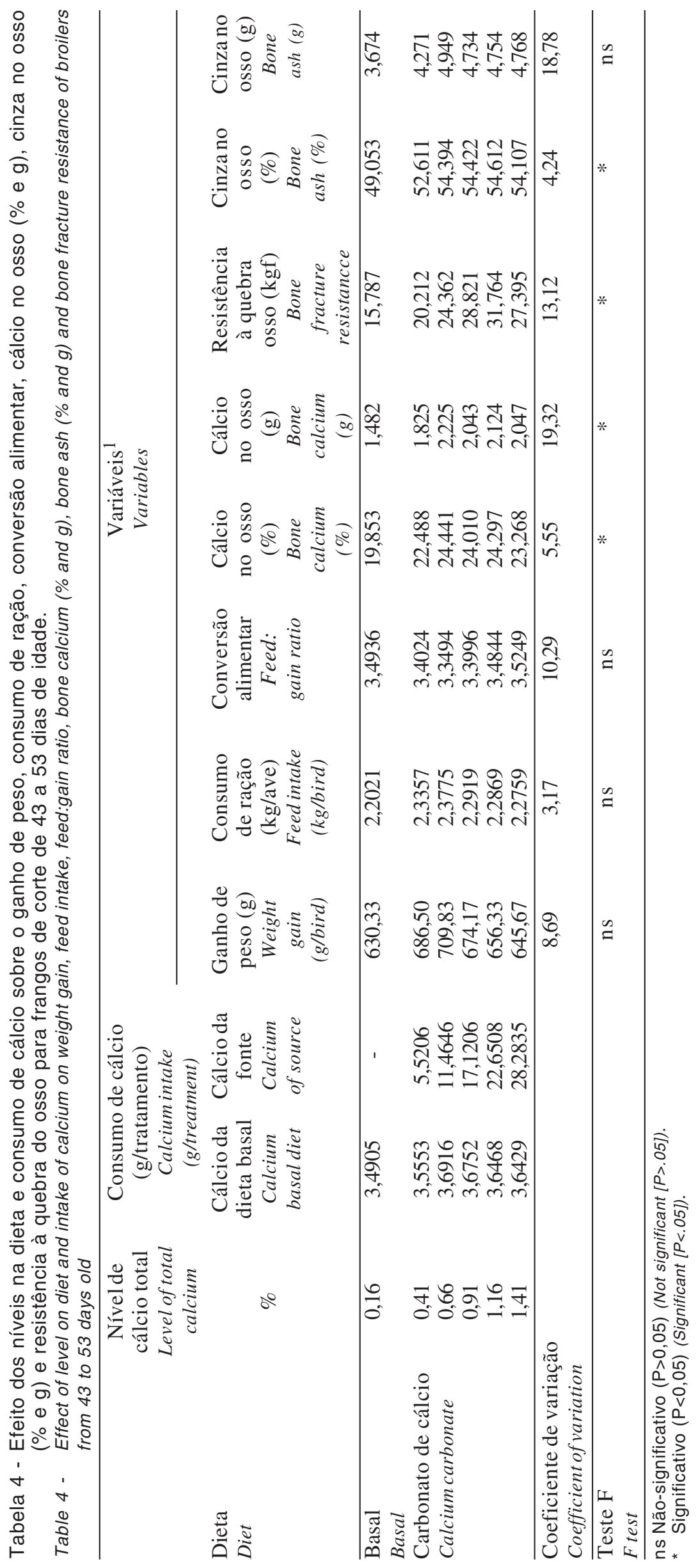


Tabela 5 - Estimativas da exigência de cálcio para frangos de corte na fase de crescimento (43 a 53 dias de idade), considerando o cálcio no osso (\% e g), a resistência à quebra dos ossos e as cinzas no osso (\%), ajustadas por meio do modelo de regressão

Table 5 - Estimates of calcium requirement for broilers in the growing phase (from 43 to 53 days old), considering bone calcium $(\%$ and $\mathrm{g})$, bone fracture resistance and bone ash (\%), adjusted by the regression models

\begin{tabular}{llll}
\hline $\begin{array}{l}\text { Modelo } \\
\text { Model }\end{array}$ & $\begin{array}{l}\text { Equação de regressão } \\
\text { Regression equation }\end{array}$ & $\begin{array}{c}\text { Pmáx/ } \\
\text { PMín }\end{array}$ & $\begin{array}{c}\text { Exigência } \\
\text { cálcio }^{1}(\%) \\
\text { Calcium }^{2} \\
\text { requirement }^{1}\end{array}$ \\
\hline
\end{tabular}

Quadrático

Quadratic

Cálcio no osso $(\%)$

Bone calcium (\%)

Cálcio no osso (g)

Bone calcium $(g)$

Resistência à quebra $(\mathrm{kgf})$

Bone fracture resistance $(\mathrm{kgf})$

Cinza no osso $(\%)$

Bone ash (\%)

$\begin{array}{llllr}\hat{Y}=17,9682+13,7439 x-7,13834 \times 2 & 24,56 & 0,91 & 0,96 * * & 0,6546204 \\ \hat{Y}=1,21925+1,92208 x-0,965257 \times 2 & 2,173 & 0,94 & 0,88^{*} & 0,0434887 \\ \hat{Y}=11,5345+26,4155 x-10,5445 \times 2 & 28,02 & 1,18 & 0,99 * & 3,168545 \\ \hat{Y}=47,068+15,5625 x-7,6243 \times 2 & 54,99 & 0,97 & 0,97 * * & 0,5786899\end{array}$

${ }_{1}^{1}$ Representa 95\% do valor máximo encontrado pela equação de regressão, indicando o nível adequado de cálcio total para cada variável. ${ }^{1}$ Represent $95 \%$ of the minimum value obtained by the regression equation, indicating the ideal total calcium levels for each levels. ** $\quad(P \leq 0,01),{ }^{*}(P \leq 0,05)$ pelo teste $F$.

Pmáx (ponto de máxima) e Pmín (ponto de mínima).

SQD - Soma de quadrados dos desvios (Sum of deviation squares).

em consideração a resistência à quebra óssea, que é uma variável importante no abate e processamento das aves, a exigência de cálcio para frangos de corte seria de 1,28 e $1,18 \%$, para as fases de crescimento e terminação, respectivamente.

\section{Literatura Citada}

ANDERSON, J.O.; DOBSON, D.C.; JACK, K. Effect of particle size of the calcium source on performance of broiler chicks fed diets with different calcium and phosphorus levels. Poultry Science, v.63, p.311-316, 1984.

BRUGALLI, I. Efeito da granulometria na biodisponibilidade de fósforo e nos valores energéticos da farinha de carne e ossos e exigência nutricional de fósforo para pintos de corte. Viçosa, MG: Universidade Federal de Viçosa, 1996. 83p. Dissertação (Mestrado em Zootecnia) - Universidade Federal de Viçosa, 1996.

CABRAL, G.H. Níveis de cálcio em rações para frango de corte. Viçosa, MG: Universidade Federal de Viçosa, 1999. 83p. Dissertação (Doutorado em Zootecnia) - Universidade Federal de Viçosa, 1999.

EDWARDS JR., H.M.; MARION, J.E.; FULLER, H.L. et al. Studies on calcium requirements of broilers. Poultry Science, v.42, p.699-703, 1963.

EDWARDS JR., H.M.; VELTMANN JR., J.R. The role of calcium and phosphorus in the etiology of tibial dyschrondroplasia in young chicks. Journal Nutrition, v.113, p.1568-1575, 1983.

EUCLYDES, R.F.; ROSTAGNO, H.S. Estimativas dos níveis nutricionais via experimentos de desempenho. In: WORKSHOP LATINO-AMERICANO AJINOMOTO

R. Bras. Zootec., v.33, n.2, p.397-406, 2004
BIOLATINA, 2001, Foz do Iguaçu. Anais... São Paulo: p.77-88, 2001.

FORMICA, S.D.; SMINT, M.J.; BACHARAC, M.M. et al. Calcium and phosphorus requirements of growing turkeys and chickens. Poultry Science, v.41 p.771-776, 1962.

GUINOTTE, F.; NYS, Y. The effects of particle size and origin of calcium carbonate on performance and ossification characteristics in broiler chicks. Poultry Science, v.70, p.1908-1920, 1991.

HULAN, H.W.; De GROOTE, G.; FOnTAINE, G. et al. The effect of different totals and rations of dietary calcium and phosphorus on the performance and incidence of leg abnormalities of male and female broiler chickens. Poultry Science, v.64, p.1157-1169, 1985.

HURWITZ, S.; PLAVNIK, I.; SHAPIRO, A. et al. Calcium metabolism and requirements of chicks are affect by growth. Journal Nutrition, v.2, p.2679-2686, 1995.

HUYGHEBAERT, G.; DE GROOTE, G.; KEPPENS, L. L'influence des teneurs en calcium et en fluor et du rapport $\mathrm{Na} / \mathrm{Cl}$ sur l'utilisation du phosphore et la solidité des os chez les poulets de chair. Revista Agrícola, v.34, p.312330, 1981

KLASING, K.C. Comparative avian nutrition. New York: Cab International, 1998. 350p.

McNAUGHTON, J.L.; DILWORTH, B.C.; DAY, E.J. Effect of particle size on the utilization of calcium supplements by the chick. Poultry Science, v.53, p.1024-1029, 1974.

KIDD, M.T.; LERNER, S.P.; ALLARD, J.P.; et al. Threonine needs of finishing broilers: growth, carcass and economic responses. Journal of Applied Poultry Research, v.8, p.160-169, 1999.

NATIONAL RESEARCH COUNCIL - NRC. Nutrients requirements of poultry. 9.ed., Washington, D.C.: 1994. $155 \mathrm{p}$.

PINHEIRO, J.W.; FONSECA, N.A.N.; MIZUBUTI, I.Y. et al. 
Efeito da retirado do calcário e/ou fosfato bicálcico da ração sobre o desempenho dos frangos de corte. In: REUNIÃO ANUAL DA SOCIEDADE BRASILEIRA DE ZOOTECNIA, 32., 1995, Brasília. Anais... Brasília: Sociedade Brasileira de Zootecnia, 1995. p.544.

ROSTAGNO, H.S.; BARBARINO JR., P.; BARBOZA, W.A. Nutrient requirement of poultry determined in Brazil. In: SIMPÓSIO INTERNACIONAL SOBRE EXIGÊNCIAS NUTRICIONAIS DE AVES E SUÍNOS, 1996, Viçosa, MG. Anais... Viçosa, MG: Universidade Federal de Viçosa, 1996. p.81-107.

ROSTAGNO, H.S.; ALBINO, L.F.T.; DONZELE, J.L. et al. Tabelas brasileiras de aves e suínos (composição de alimentos e exigências nutricionais). 1.ed. Viçosa, MG: Universidade Federal de Viçosa, 2000. 141p.

RUNHO, R.C.; GOMES, P.C.; ROSTAGNO, H.S. et al. Exigência de fósforo disponível para frangos de corte machos e fêmeas. Revista da Sociedade Brasileira de Zootecnia, v.30, n.1, p.187-196, 2001.

SCHEIDELER, S.E.; RIVES, D.V.; GARLICH, J.D. et al. Dietary calcium and phosphorus effects on broiler performance and the incidence of sudden death syndrome mortality. Poultry Science, v.74, p.2011-2018, 1995.

SILVA, D.J. Análise de alimentos (métodos químicos e biológicos). 2.ed. Viçosa, MG: Universidade Federal de Viçosa, 1998. 165p.

SIMCO, T.F.; STEPHENSON, E.L. Reevaluation of the calciumphosphorus requirements of the chick. Poultry Science, v.40, p.1188-1192, 1961.

SKINNER, J.T.; IZAT, A.L.; WALDROUP, P.W. Effects of removal of supplemental calcium and phosphorus fromin broiler finisher diets. Journal of Applied Poultry Research, v.1, n.3, p.42-47, 1992.
SKINNER, J.T.; WALDROUP, P.W. Effects of calcium and phosphorus levels in starter and grower diets in broiler during the finisher period. Journal of Applied Poultry Research, v.1, n.3, p.277-279, 1992.

SMITH, O.B.; KABAIJA, E. Effect of high dietary calcium and wide calcium-hosphorus rations in broiler diets. Poultry Science, v.64, p.1713-1720, 1984.

TWINING, P.F.; LILLIE, E.J.; ROBEL, E.J. et al. Calcium and phosphorus requirements of broiler chickens. Poultry Science, v.43, p.283-296, 1964.

UNIVERSIDADE FEDERAL DE VIÇOSA - UFV. Central de Processamento de Dados - UFV/CPD. SAEG - Sistema para análise estatística e genética, versão 7.0 Viçosa, MG: Universidade Federal de Viçosa, 1997. 54p.

WALDROUP, P.W.; AMMERMAN. B.C.; HARMS, R.H. Comparison of the requirements of battery and floor-grown chicks for calcium and phosphorus. Poultry Science, v.41, p.1433-1436, 1962.

WALDROUP, P.W.; MITCHELL, R.J.; HAZEN, K.R. The phosphorus needs of finishing broilers in relationship to dietary nutrient density levels. Poultry Science, v.53, p.1655-1663, 1974.

WHITE-STEVENS, E.R.; PENSACK, J.M.; STOKSTAD, E.L.R. The calcium and phosphorus requirement of the chick. Poultry Science, v.39, p.1305, 1960.

YOSHIDA, M.; HOSHII, H. Re-avaluation of requirement of calcium and available phosphorus for starting meat-type chicks. Japanese Poultry Science, v.19, p.101-109, 1982.

Recebido em: 20/06/02

Aceito em: 07/04/03 